\title{
Efficient Opportunistic Network Creation in the Context of Future Internet
}

\author{
Andreas Georgakopoulos, Kostas Tsagkaris, Vera Stavroulaki, and \\ Panagiotis Demestichas \\ University of Piraeus, Department of Digital Systems, \\ 80, Karaoli and Dimitriou Street, 18534 Piraeus, Greece \\ \{andgeorg, ktsagk, veras, pdemest\} @unipi.gr
}

\begin{abstract}
In the future internet era, mechanisms for extending the coverage of the wireless access infrastructure and service provisioning to locations that cannot be served otherwise or for engineering traffic whenever the infrastructure network is already congested will be required. Opportunistic networks are a promising solution towards this direction. Opportunistic networks are dynamically created, managed and terminated. During the creation phase, nodes that will constitute the opportunistic network needs, are selected and assigned with the proper spectrum and routing patterns. Accordingly, this paper focuses on the opportunistic network creation problem and particularly on the efficient selection of nodes to participate therein. A first step towards the formulation and solution of the opportunistic network creation problem is made, whereas indicative results are also presented in order to obtain some proof of concept for the proposed solution.
\end{abstract}

Keywords: Opportunistic Networks, Node Selection, Coverage Extension, Capacity Extension, Future Internet.

\section{$1 \quad$ Introduction}

The emerging wireless world will be part of the Future Internet (FI). All kinds of devices and networks will have the interconnection potential. Thus, any object or network element will have communication capabilities embedded and several objects in a certain environment will be able to create a communication network. Challenges such as the infrastructure coverage extension or the infrastructure capacity extension, arise.

Opportunistic networking seems a promising solution to the problem of coverage extension of the infrastructure in order to provide service to nodes which normally would be out of the infrastructure coverage or to provide infrastructure decongestion by extending its capacity. In general, opportunistic networks (ONs) involve nodes and terminals which engage occasional mobility and dynamically configured routing patterns. They can comprise numerous network elements of the infrastructure, and terminals/ devices potentially organized in an infrastructure-less manner. It is assumed that 
ONs are operator-governed, coordinated extensions of the infrastructure in order to assist the infrastructure and not to be used as an alternative to the infrastructure. Operator governance of ONs is achieved through policies provided by the operator network management. The network management provides the overall operational framework of the ON but it is out of the scope of this work.

Further on, ONs will exist temporarily, i.e., for the time frame necessary to support particular network services and accommodate new FI-enabled applications (requested in a specific location and time). A region could be served by more than one ONs that could co-exist, under the coordination of the operator. At the lower layers, the operator designates the spectrum that will be used for the communication of the nodes of the $\mathrm{ON}$ (i.e., the spectrum derives through coordination with the infrastructure). On the other hand, the network layer capitalizes on context, policy, profile, and knowledge awareness to optimize routing and service/ content delivery. Additionally, mechanisms for the efficient, dynamic creation of ONs are needed to be developed which should comply node selection according to specific, predefined characteristics.

To that respect, ONs can be seen as part of the Cognitive Control Network (CCN) as illustrated in Fig. 1 and is proposed by ETSI in [1]. The CCN involves an emerging group of functionalities aiming at introducing cognition mechanisms to the evolving wireless world. The spontaneous creation of ONs can comprise the outcome of advanced decision making provided by such cognitive mechanisms. Framed within this statement, this work discusses on the $\mathrm{ON}$ creation as a means to provide extended coverage to the infrastructure and/or deplete congested parts of it, and in particular, it introduces a node selection algorithm and evaluates its effectiveness by means of simulation.

The rest of the article is structured as follows: the second section discusses the related work in the area of node selection and coexistence of ONs with infrastructure elements. Third section discusses the high-level solution of the opportunistic networking concept and defines the phases through the lifecycle of the ON (i.e. ON suitability determination, ON creation, ON maintenance and ON termination). The fourth section focuses on the $\mathrm{ON}$ creation phase and provides the algorithmic solution of the opportunistic node selection problem statement with respect to indicative scenarios such as the opportunistic coverage extension or the opportunistic capacity extension. The fifth section provides simulation result sets in order to evaluate the proposed algorithm and strengthen the proof of concept. Finally, the article concludes with key findings and future work.

\section{Related Work}

Various approaches concerning node selection for wireless sensor or mesh networks have been already discussed. For example, random node selection in unstructured P2P networks is discussed in [2] while authors in [3] address the relay selection problem in cooperative multicast over wireless mesh networks. Also, in [4], analytical and simulation approaches are used in order to investigate the relationship between the lifetime of sensor networks and the number of reporting nodes and to provide the trade-off between maximizing network lifetime and the fastest way to report an event in a wireless sensor node. 


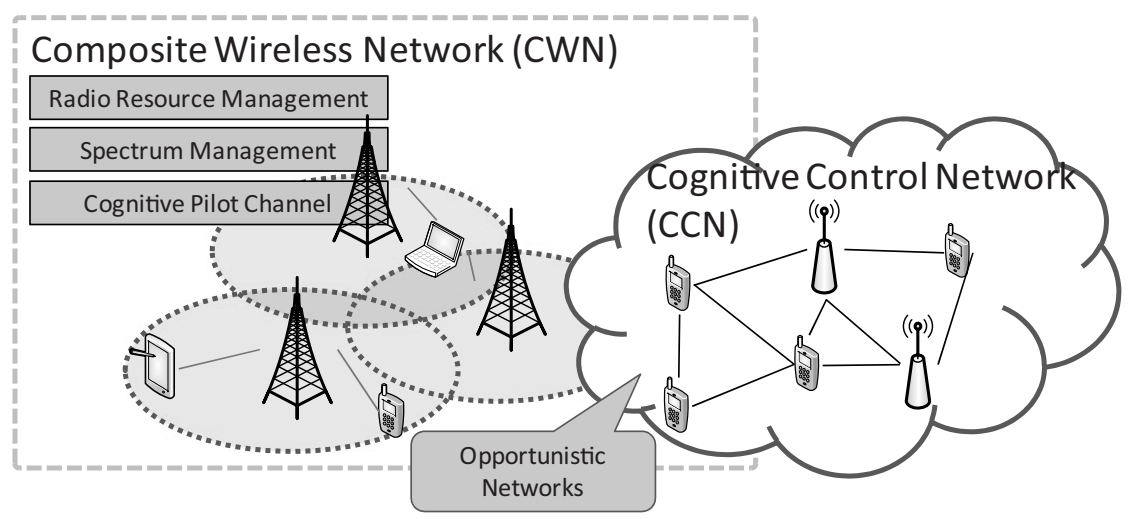

Fig. 1. The emerging cognitive wireless world

In [5], the selection and navigation of mobile sensor nodes is investigated by taking into consideration three metrics including coverage, power and distance of each node from a specified area. In [6], a grid-based approach for node selection in wireless sensor networks is analyzed, in order to select as few sensors as possible to cover all sample points. In [7], the issue of server selection is being investigated by proposing a node selection algorithm with respect to the worst-case link stress (WLS) criterion. These works are proposing specific sensor node selection algorithms by taking into consideration attributes such as the area of coverage, the navigation/ mobility issues of moving sensors, or the minimization of the number of relays.

Despite the fact that ONs may resemble mesh networking or ad hoc networking, certain differences do exist. For example, mesh networking is not used for the expansion of the coverage of the infrastructure, but for the wireless coverage of an area using various Radio Access Technologies (RATs) [8]. Hence, they are not operator-governed. Moreover, ad hoc networking uses peer nodes to form an infrastructure-less, self-organized network [9], but does not necessarily have the logic of specific node selection for the efficient network creation according to a pre-specified set of parameters. With the proposed ON approach, issues like these and limitations of ad hoc and mesh networking are trying to be addressed and resolved. ON comes with a bundle of benefits that could evolve in areas where infrastructure is difficult to exist or communication demand rises instantly and support is needed for successful handling.

Also, authors in [10] propose and implement a Virtual Network Service (VNS) as a value-added network service for the deployment of Virtual Private Networks (VPNs) in a managed wide area IP network while in [11] a survey regarding automatic configuration of VPNs takes place where auto-configuration mechanisms are discussed and compared. Virtual network provisioning across multiple substrate networks is studied in [12] where authors evaluate the resource matching, splitting, embedding and binding steps required for virtual network provisioning. However, Virtual Networks (VNs) such as VPNs intend to be used among public infrastructures to provide secure, remote access to users of e.g. an office network. Also, Virtual LANs (VLANs) which are another type of VNs are logical networks which are based on physical net- 
works and can be used for grouping of hosts in the same domain regardless of their physical location. Compared to the proposed ON approach, it is clear that VNs may be temporarily established (e.g. a VPN which is established between home and office network for a certain amount of time) but they are not operator-governed nor are they coordinated extensions to places where infrastructure is not available.

To that respect, the contribution of this work is to propose a unified solution for ON creation which takes into consideration the dynamic nature of such networks and the application provisioning via the use of various kinds of nodes (e.g. cell phones, PDAs, laptops and other network-enabled devices). Thus, a fitness function is presented which is able to evaluate the eligibility of each candidate node and accept it or reject it from participating to the $\mathrm{ON}$.

\section{Solution Approach Based on ONs}

The proposed ON approach is based on four discrete phases. These phases include the ON suitability determination, the ON creation, the ON maintenance and the ON termination. To that respect, each phase is previewed in the following subsections.

\subsection{ON Suitability Determination}

Specifically, the suitability determination phase is rather crucial, because based on the observed radio environment, the node capabilities, the network operator policies and the user profiles, the outcome of this phase will be to decide whether it is suitable to set-up an operator-governed $\mathrm{ON}$ or not, at a specific time and place. In order to evaluate the suitability, the detection of opportunities for ON establishment with respect to total nodes and potential radio paths should be taken into consideration as main inputs. As a result, the network operator needs to be aware (by discovery procedures) of the nodes' related information.

Each node is distinguished by a set of characteristics. Node characteristics will include the capabilities (including available interfaces, supported RATs, supported frequencies, support of multiple connections, relaying/bridging capabilities) and status of each candidate node in terms of resources for transmission (status of the active links), storage, processing and energy. Moreover, the operator needs to be aware of the location and the mobility level of each node. A prerequisite of each case (e.g. opportunistic coverage extension or opportunistic capacity extension) is that the nodes need to have some type of access to the infrastructure, or to have some type of access to a decongested Access Point (AP).

Furthermore, application requirements and the similarity level of the requested applications (i.e. common application interests) have to be taken into consideration by defining the involved applications, their resource requirements, and their appropriateness for being provided through ONs. Also, the potential gains from a possible ON creation should be considered in order to provide the main output of this phase which will be the request for $\mathrm{ON}$ creation. 


\subsection{ON Creation}

The next phase of the ON lifecycle is the ON creation. The ON creation phase is responsible for providing mechanisms and decisions for effectively creating the ON based on the output received from the suitability determination phase. It focuses on selecting participant nodes according to the previously mentioned set of characteristics and on choosing optimal radio paths in order to ensure optimal QoS. Finally, it performs all the required procedures to effectively connect $\mathrm{ON}$ members with each other and to ensure continuity of service for the members with regard to the infrastructure. The main output of the ON creation phase will be the selected nodes and the selected links/interfaces/RATs/spectrum.

\subsection{ON Maintenance}

Once the $\mathrm{ON}$ is created, it will have to adapt dynamically during all its operational lifetime to changing environment conditions (e.g. context, operator's policies, user profiles). In order to achieve this, after the successful completion of the creation phase, the maintenance phase has to be initiated. Generally, the maintenance phase will have to monitor nodes, spectrum, operator's policies, QoS on a frequent basis and to decide whether it is suitable to proceed to a reconfiguration of the ON. Thus, the maintenance phase is responsible for the opportunistic network management and reconfiguration. Monitoring mechanisms during the operation of the $\mathrm{ON}$ would be introduced in order to accommodate any alterations that are made in the $\mathrm{ON}$ and act accordingly.

\subsection{ON Termination}

The termination phase will eventually take the decision to release the ON, thus triggering all the necessary procedures and associated signaling. It is distinguished according to the reason of termination. As a result, there may be termination of the $\mathrm{ON}$ due to cessation of application provision, termination due to inadequate gains from the usage of the $\mathrm{ON}$ and forced termination.

Fig. 2 illustrates the previously mentioned phases and their possible interconnections. According to this, the suitability determination phase issues a creation request which triggers the $\mathrm{ON}$ creation. Once the $\mathrm{ON}$ is successfully created, frequent monitoring is needed in order to maintain the flawless and high-quality operation of the $\mathrm{ON}$. If needed, reconfiguration procedures may take place by re-creating the $\mathrm{ON}$. Moreover, the maintenance phase may issue a termination request in the cases where the ON experiences sudden and unsolved drop in QoS, or when the application provision has successfully ended, thus the ON is not needed anymore. 


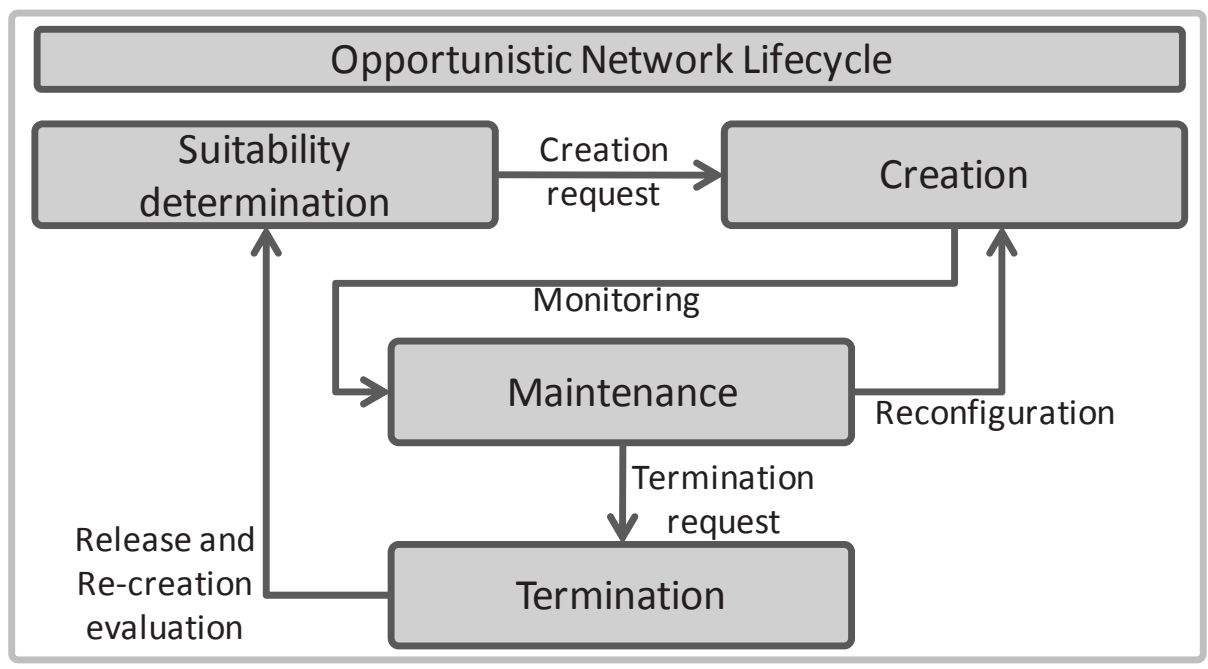

Fig. 2. The ON Lifecycle

\section{Opportunistic Network Creation}

Following the ON lifecycle overview solution, this work focuses on the part of the ON creation phase and more specifically to the selection of nodes which will form the $\mathrm{ON}$. In order to be able to create the $\mathrm{ON}$ and enable service provisioning to end-users, it is needed to gain awareness of the status of candidate, relay nodes (i.e. nodes that can be used as routers, even when they do not need to use an application) and the application nodes (i.e. the nodes that use a specific application). Moreover, gateway nodes have to be defined in order to provide connectivity between the $\mathrm{ON}$ and the infrastructure (e.g. Macro Base Station -MBS), upon request.

To that respect, some indicative business scenarios have been identified in order to elaborate on the ON paradigm. Fig. 3 illustrates the opportunistic coverage extension scenario. According to this scenario, a node which acts as a traffic source like a laptop or a camera is out of the coverage of the infrastructure. As a result, a solution would comprise the creation of an $\mathrm{ON}$ in order to serve the out of infrastructure coverage node. Opportunism primarily lies in the selection for participation in the $\mathrm{ON}$ of the appropriate subset of nodes, among the candidate nodes that happen to be in the vicinity, based on profile and policy information of the operator and the use of spectrum that will be designated by the network operator, for the communication of the nodes of the ON. Through the opportunistic approach multiple benefits for key players derive such as, the end user gets access to the infrastructure in situations where it normally would not be possible, while the access provider may experience increased cashflow as more users are being supported.

Another indicative scenario would comprise the notion of the opportunistic capacity extension. According to this scenario, a specific area experiences traffic congestion issues and an $\mathrm{ON}$ is created in order to route the traffic to non-congested APs. 
Access providers are benefited from the fact that more users can be supported since new incoming users that otherwise would be blocked can now be served, while end users experience improved QoS since congestion situations can be resolved as illustrated in Fig. 4.

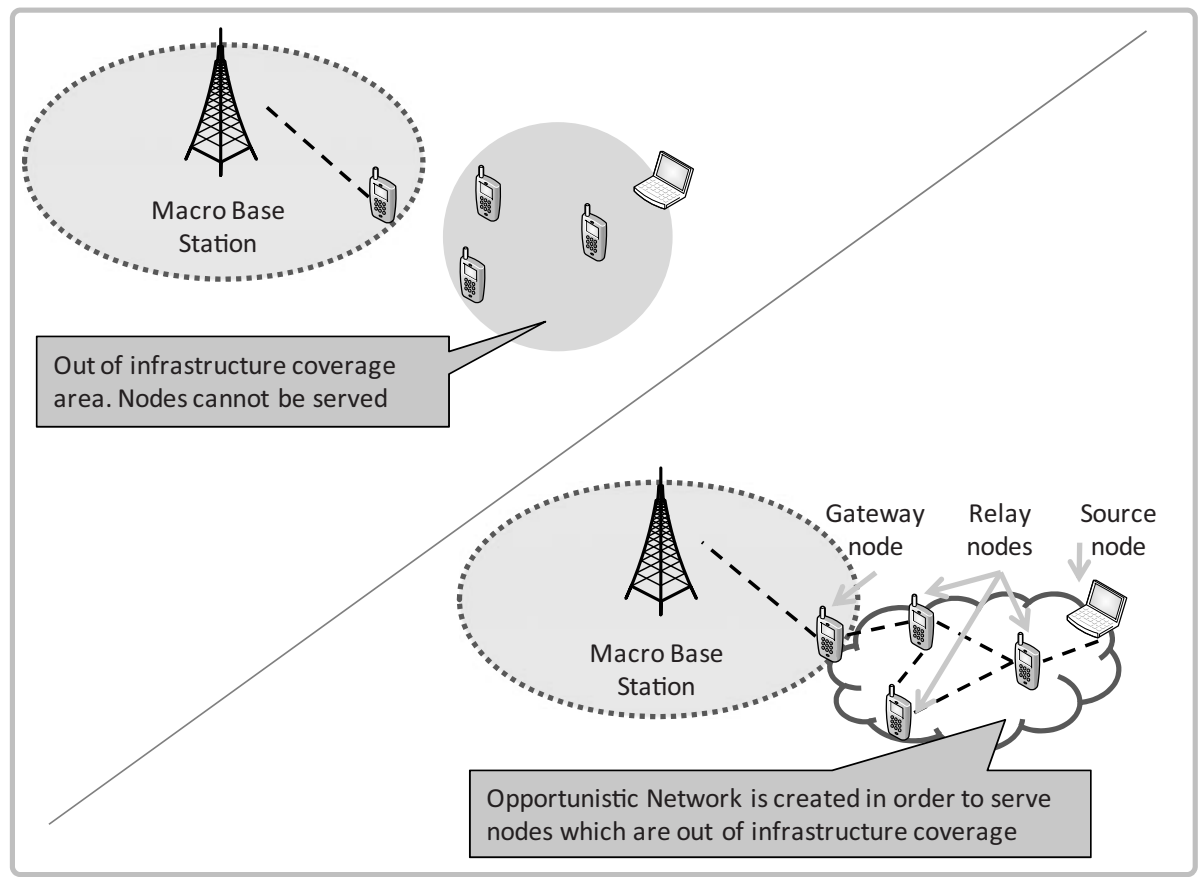

Fig. 3. Opportunistic coverage extension scenario. An ON is created in order to serve the out of infrastructure coverage nodes.

To that context, in order to gain awareness of the status of the candidate nodes in the vicinity, a monitoring mechanism is needed, that will be able of monitoring aspects that has to take into account, each node's related information in order to be able to calculate a fitness function. The monitored aspects are:

- Energy level of the node

- Availability level of the node -taking into account node's capabilities (including available interfaces, supported RATs, supported frequencies, support of multiple connections, relaying/ bridging capabilities, gateway capabilities-wherever applicable), status of each node in terms of resources for transmission (status of the active links), storage, processing, mobility levels -location, supported applications (according to node capabilities and application requirements).

- Delivery probability of the node 


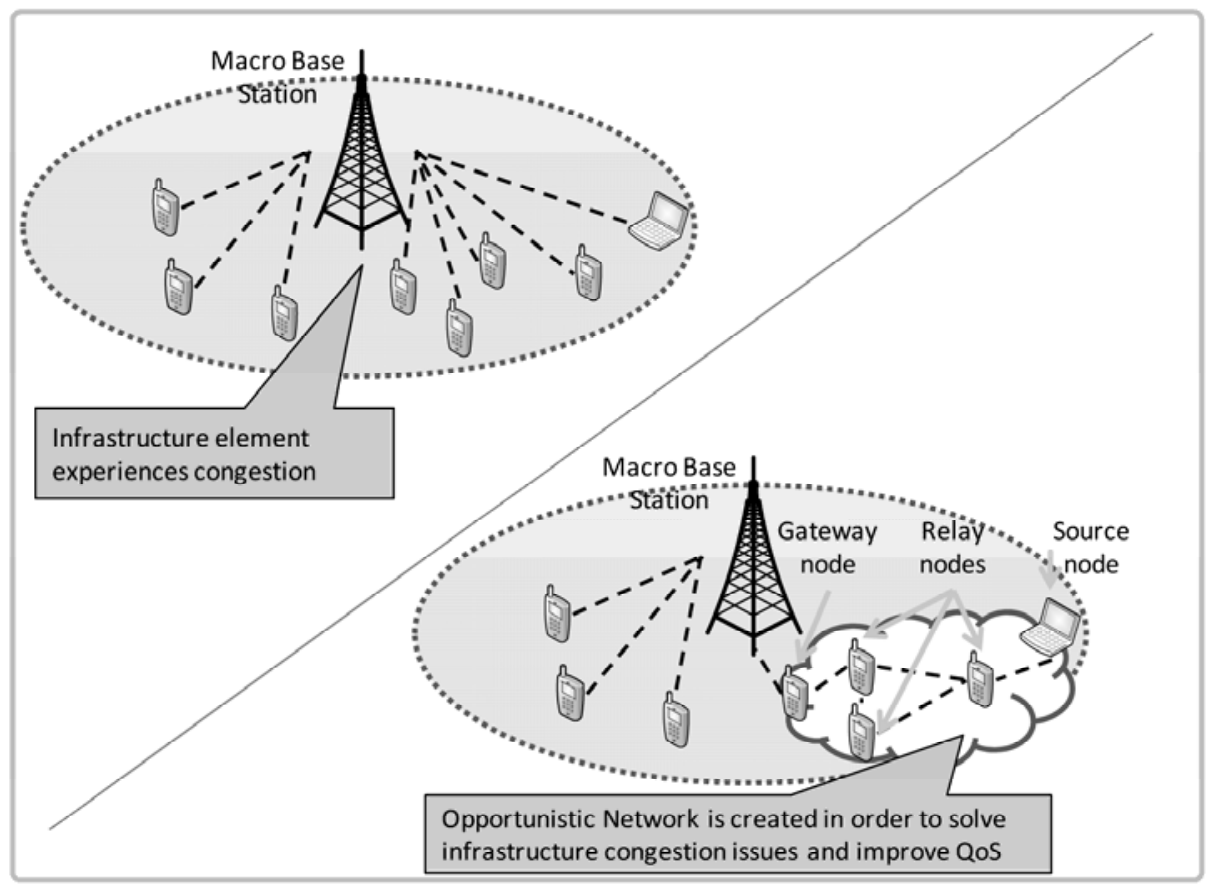

Fig. 4. Opportunistic capacity extension scenario. An ON is created in order to alleviate congestion in the infrastructure and improve QoS.

The aforementioned node characteristics provide the input to a fitness function which according to a specified threshold, decides on whether to accept or reject a candidate node for being part of the ON. The accepted nodes are eligible of forming the ON. Relation (1) below shows the fitness function considered in this paper for the node selection:

Fitness Function $=x_{i} *\left[\left(e_{i} * w_{e}\right)+\left(a_{i} * w_{a}\right)+\left(d_{i} * w_{d}\right)\right]$.

where $e_{i}$ denotes the energy level of node $i, a_{i}$ denotes the availability level of node $i$ at a specific moment and $d_{i}$ denotes the delivery probability of packets of node $i$. Also $x_{i}$ acts as multiplier according to Relation (2):

$x_{i}=\left\{\begin{array}{l}1, e_{i}>0 \cap a_{i}>0 \\ 0, e_{i}=0 \cup a_{i}=0\end{array}\right.$.

On the other hand, for the definition of weights of the fitness function, the Analytic Hierarchy Process (AHP) has been used. As the AHP theory suggests [13], initially a decision maker decides the importance of each metric as AHP takes into account the decision maker's preferences. For our example it is assumed that energy is a bit more important than availability and the delivery probability is less important from both energy and availability. According to these assumptions a matrix is completed. The 
matrix contains the three factors (i.e. energy, availability and delivery probability) along with their levels of importance. As a result, the weight for energy is $w_{e}=0.53$, the weight for availability is $w_{a}=0.33$ and the weight for delivery probability is $w_{d}=0.14$. Furthermore, the theory explains that in order to have consistent results an $\lambda_{\max }$ attribute must be greater than the absolute number of the proposed factors (i.e. 3 for our assumption). The derivation of the $\lambda_{\max }$ attribute is also explained in the AHP technique [13] and it is used for the checking of sanity of the weights. In our example the $\lambda_{\max }$ turns to be 3.05 , hence the derived results are on safe ground. Finally, a Consistency Ratio is calculated. Saaty in [13] argues that a Ratio $>0.10$ indicates that the judgments are at the limit of consistency though Ratios $>0.10$ could be accepted sometimes. In our case, the Consistency Ratio is around 0.04, well below the 0.10 threshold, so our estimations tend to be trustworthy according to the AHP theory.

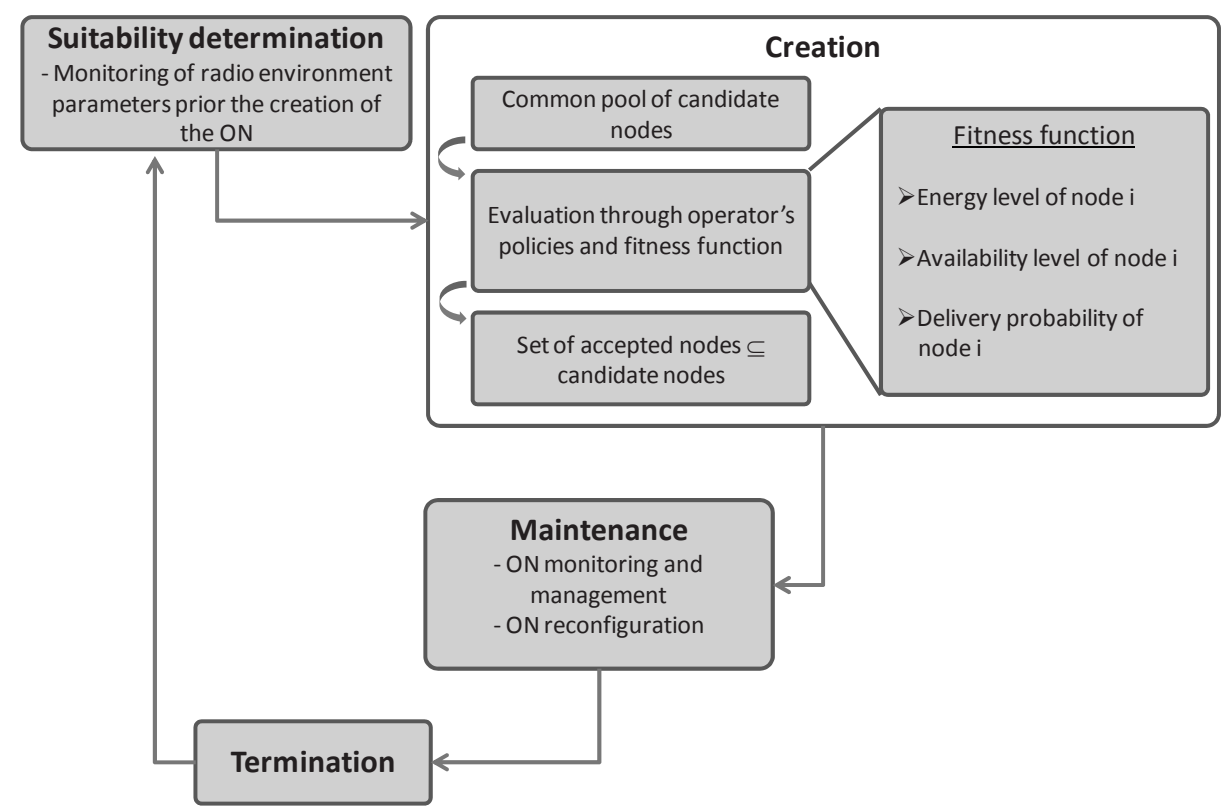

Fig. 5. Detailed view of the ON creation phase

Additionally, the detailed view of the ON creation phase is depicted in Fig. 5 where the responsibilities of the creation phase are visually explained. These responsibilities include the evaluation and selection of nodes and spectrum conditions which will provide the set of the accepted nodes to the ON. Also, the interconnections of the creation phase with the suitability determination and the maintenance are visible.

\section{$5 \quad$ Results}

In order to obtain some proof of concept for our network creation solution, a Javabased prototype has been developed which calculates the fitness function and informs 
the system on the accepted and rejected nodes. By using the derived number of accepted nodes, indicative results on the potential performance of these nodes when used in an ON have been also collected and analyzed using the Opportunistic Network Environment (ONE) [14], [15].

An indicative network topology of 60 total participant non-moving nodes is illustrated in Fig. 6. Each node features 2 interfaces, a Bluetooth (IEEE 802.15.1) [16] and a high-speed interface (e.g. IEEE 802.11 family [17]). According to the high-speed interface, each node has a transmission data rate of $15 \mathrm{Mbps}$. On the other hand, the Bluetooth interface has a transmission data rate of $1 \mathrm{Mbps}$ but it is used for a rather short-range coverage (e.g. 10 meters). Also, every new message is created at a 30second interval and has a variable size ranging from 500 to 1500 kilobytes, depending on the scenario. Messages are created only from specific 3 hosts which are acting as source nodes, and are transmitted to specific 3 nodes which are acting as destination nodes via all the other relay nodes. Moreover, the initial energy level of each node is not fixed for all nodes, but it may range from 20 to 90 percent of available battery.

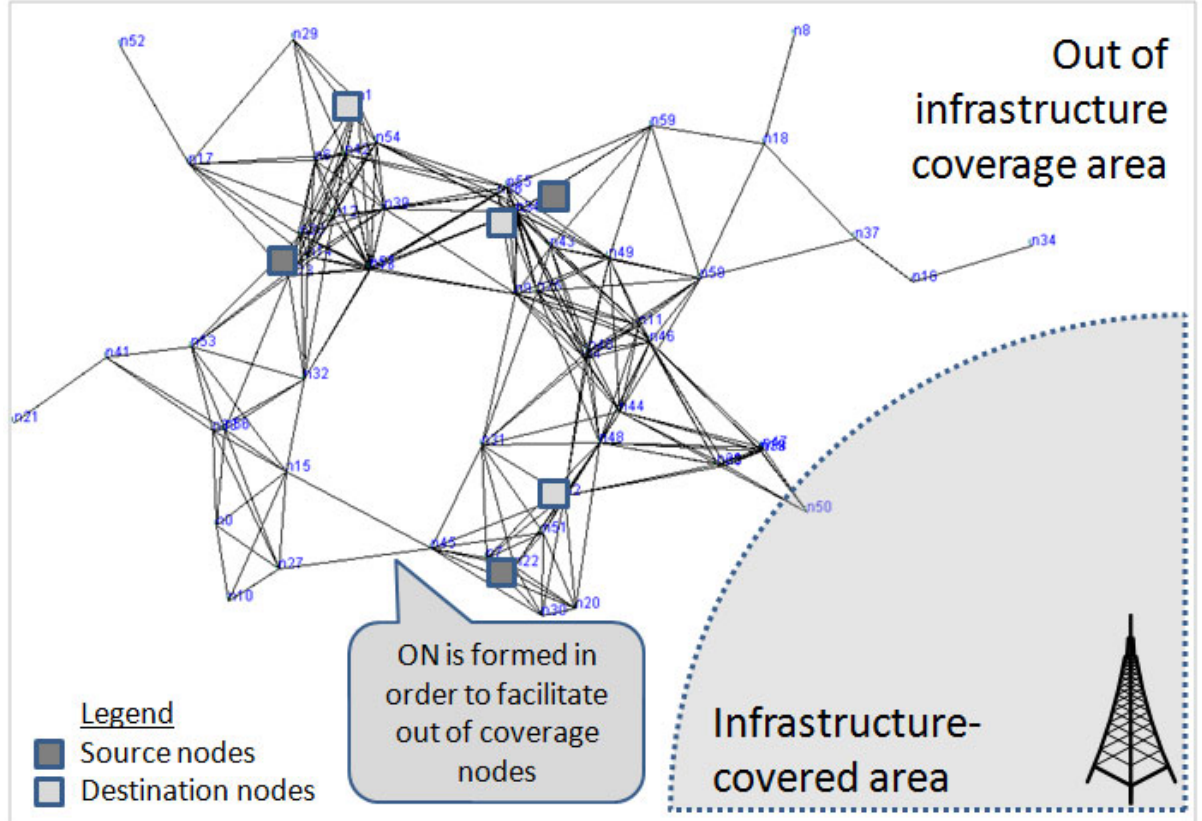

Fig. 6. Indicative network topology of 60 total nodes consisting of 3 source nodes, 3 destination nodes and 54 relay nodes

Fig. 7 shows results from the ONE simulator of the delivery probability for a variable value of nodes, ranging from 7 to 120 nodes. The routing protocol that was used for the specific simulation was the Spray \& Wait protocol [18]. According to the observations, when the 500 kilobytes fixed message was used, higher delivery rates were observed for every number of accepted nodes. On the other hand, there is a tendency 
of significantly lower delivery rates as the message size increases to 1000 and 1500 kilobytes. Moreover, the results are compared with a simulation that ran for a variable message size ranging from 500 to 1000 kilobytes as illustrated in Fig. 8. So, it seems that as the message size is increased the delivery rates tend to decrease.

Fig. 9 compares the maximum lifetime of the $\mathrm{ON}$ with the expected delivery probability of the variable message size scenario (from 500 to 1000 kilobytes). The maximum lifetime of the ON corresponds to the last link that is expected to drop during the simulation. It is shown that as the ON expands (i.e. the number of accepted nodes according to the fitness function increases), the lifetime of the ON tends to reach a maximum level and then keep stable. On the other hand, as the ON expands, the observed delivery probability of messages tends to decrease.

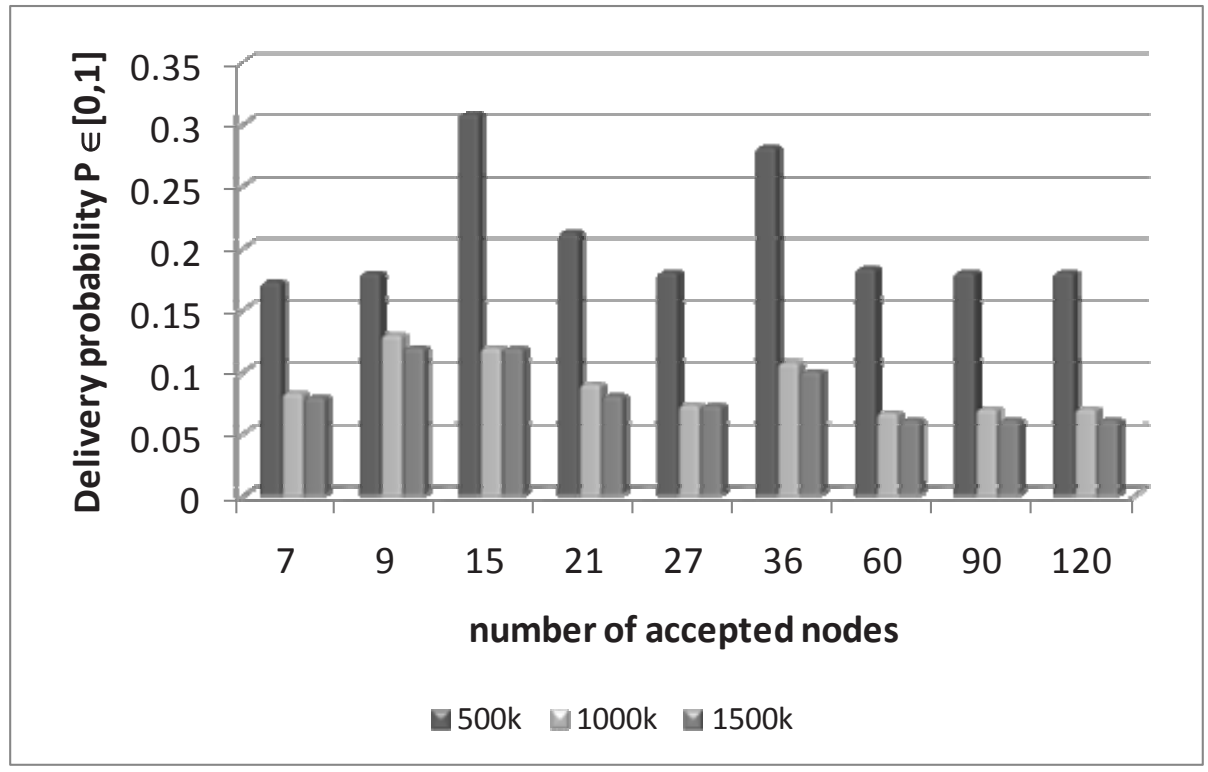

Fig. 7. Delivery probability rates for fixed message sizes ranging from 500 kilobytes to 1500 kilobytes

Also, Fig. 10 illustrates the average number of hops in the vertical axis and the number of nodes in the horizontal axis for 500, 1000, 1500 kilobytes of fixed message sizes and the variable message size ranging from 500 to 1000 kilobytes. In this chart, the tendency of increment of the average number of hops as the number of nodes increases is clear. The average number of hops corresponds to the summation of each number of hops observed by each accepted node divided by the actual number of accepted nodes. 


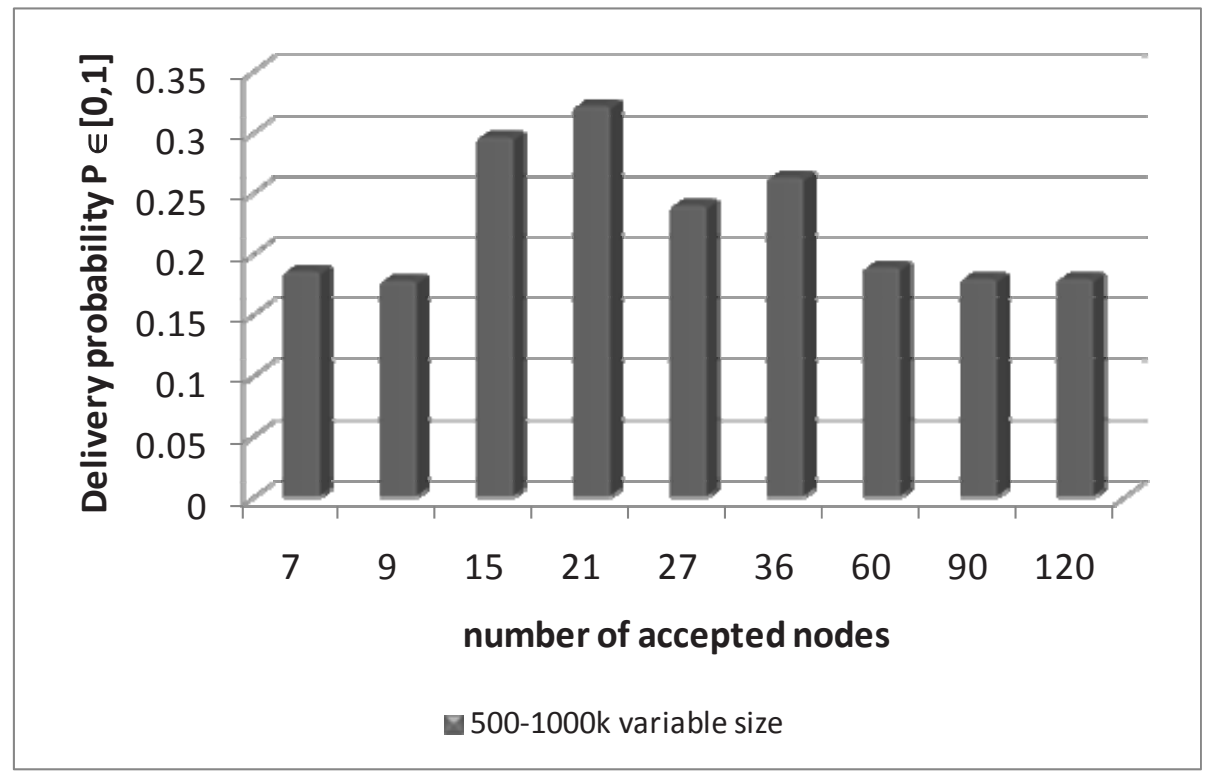

Fig. 8. Delivery probability rates for variable message sizes ranging from 500 kilobytes to 1000 kilobytes

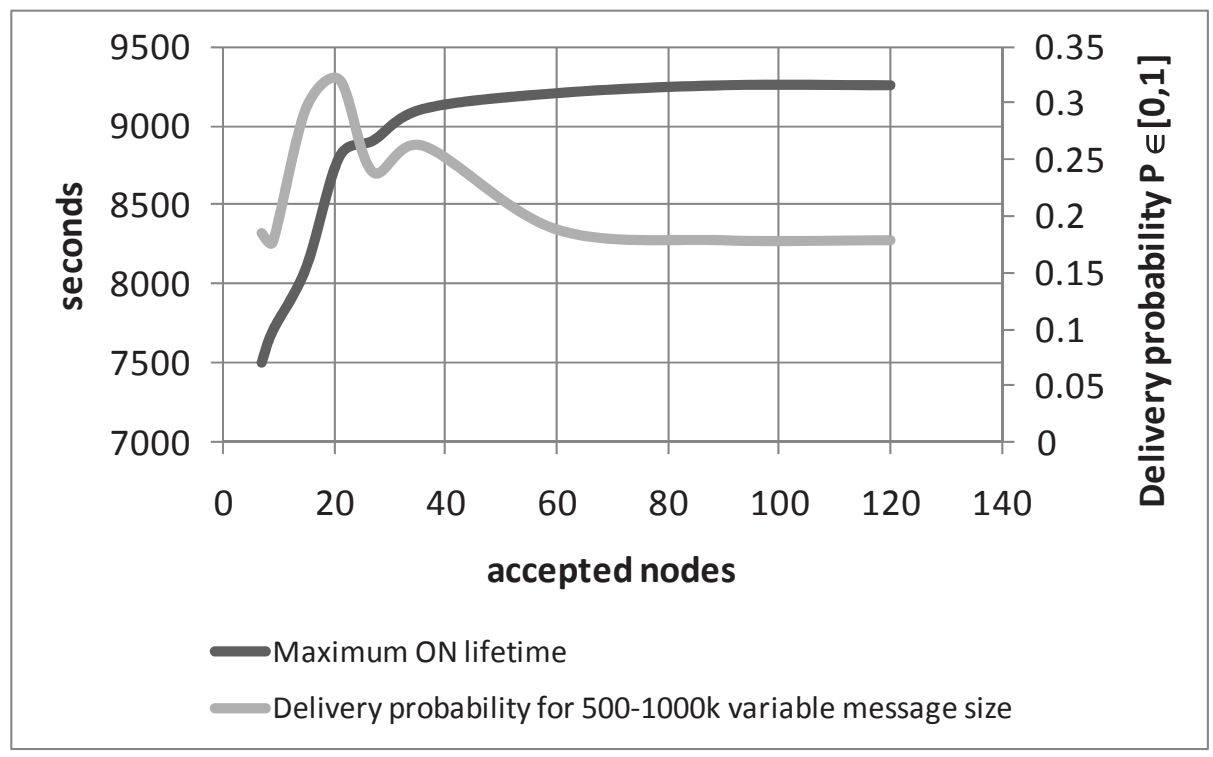

Fig. 9. Delivery probability rates compared to maximum network lifetime for a variable number of nodes ranging from 7 to 120 and a variable message size ranging from 500 kilobytes to 1000 kilobytes 


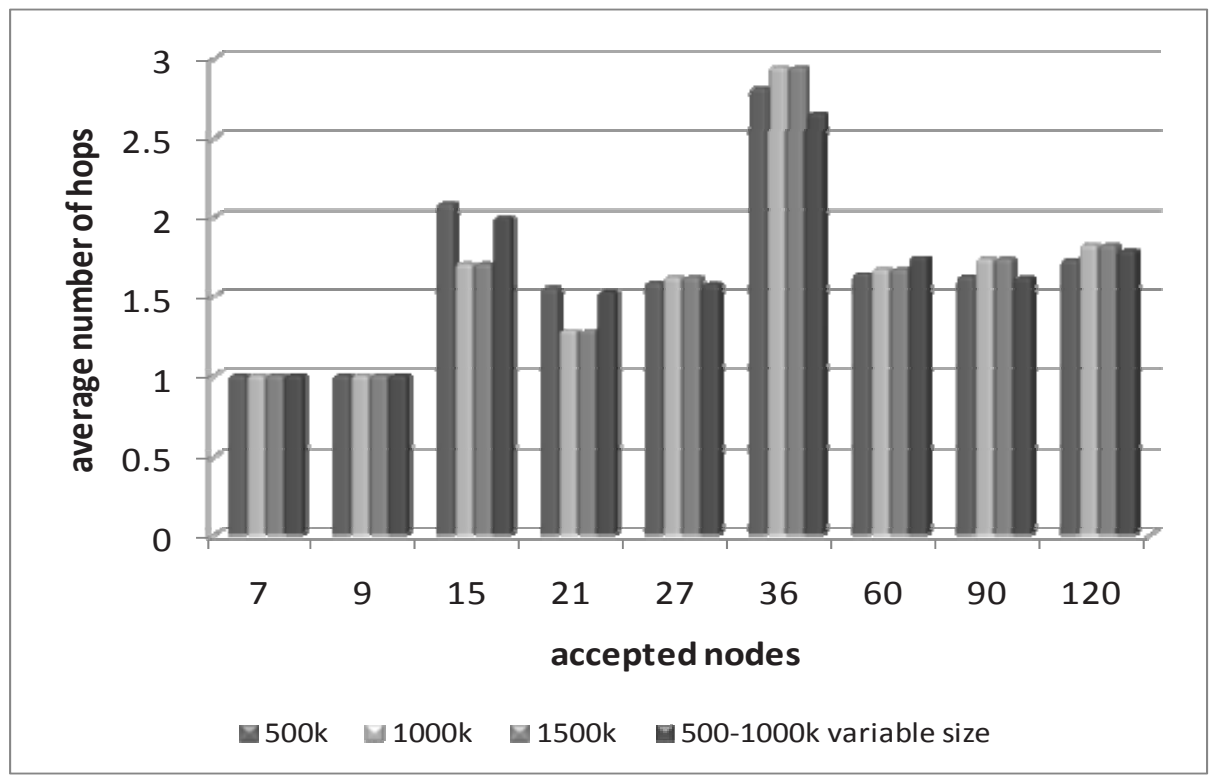

Fig. 10. Average number of hops for a variable number of nodes ranging from 7 to 120

\section{Conclusion and Future Work}

This work presents the efficient $\mathrm{ON}$ creation in the context of Future Internet. Operator-governed ONs are a promising solution for the coverage or capacity extension of the infrastructure by providing extra coverage or capacity wherever and whenever needed without the operator having to invest to expensive infrastructure equipment in order to serve temporary load surge in an area.

For the efficient creation of the $\mathrm{ON}$, specific node attributes need to be taken into consideration in order to ensure flawless application streams. As a result, participant nodes are not chosen randomly but according to a set of evaluation criteria as proposed to this work. Nevertheless, according to the provided simulation result sets, it is shown that as the message size increases from 500 to 1500 kilobytes the delivery rates tend to decrease. This is also compared to the fact that the network lifetime according to the ONE simulator, is not increased from a specific number of nodes and above as it tends to reach the maximum level and stabilize after around 60 nodes. Finally, the average number of hops tends to increase as the number of nodes increases.

Future plans include the development of algorithmic approaches for the post-creation phases of the ON (i.e. the maintenance and the termination) in order to provide cognitive ON monitoring and reconfiguration mechanisms and handle successfully the normal or forced $\mathrm{ON}$ termination situations. ON management procedures are part of the post-creation phase (i.e. the maintenance) and as a result are a subject of future study.

Acknowledgment. This work is performed in the framework of the European-Union funded project OneFIT (www.ict-onefit.eu). The project is supported by the European Community's Seventh Framework Program (FP7). The views expressed in this docu- 
ment do not necessarily represent the views of the complete consortium. The Community is not liable for any use that may be made of the information contained herein.

Open Access. This article is distributed under the terms of the Creative Commons Attribution Noncommercial License which permits any noncommercial use, distribution, and reproduction in any medium, provided the original author(s) and source are credited.

\section{References}

1. European Telecommunications Standards Institute (ETSI), Reconfigurable Radio Systems (RRS), "Summary of feasibility studies and potential standardization topics", TR 102.838, V.1.1.1 (October 2009)

2. Vishnumurthy, V., Francis, P.: On Heterogeneous Overlay Construction and Random Node Selection in Unstructured P2P Networks. In: INFOCOM 2006, 25th IEEE International Conference on Computer Communications (2006)

3. Rong, B., Hafid, A.: A Distributed Relay Selection Algorithm for Cooperative Multicast in Wireless Mesh Networks. In: 5th International Conference on Mobile Ad-hoc and Sensor Networks, Fujian (2009)

4. Bouabdallah, F., Bouabdallah, N.: The tradeoff between maximizing the sensor network lifetime and the fastest way to report reliably an event using reporting nodes' selection. Computer Communications 31, 1763-1776 (2008)

5. Verma, A., Sawant, H., Tan, J.: Selection and navigation of mobile sensor nodes using a sensor network. Pervasive and Mobile Computing 2, 65-84 (2006)

6. Chen, H., Wu, H., Tzeng, N.: Grid-based Approach for Working Node Selection in Wireless Sensor Networks. In: IEEE International Conference on Communication (2004)

7. Han, S., Xia, Y.: Optimal node-selection algorithm for parallel download in overlay content-distribution networks. Computer Networks 53, 1480-1496 (2009)

8. Akyildiz, I., Wang, X., Wang, W.: Wireless mesh networks: a survey. Computer Networks 47, 445-487 (2005)

9. Akyildiz, I., Lee, W., Chowdhury, K.: CRAHNs: Cognitive radio ad hoc networks. Ad Hoc Networks 7, 810-836 (2009)

10. Lim, L.K., Gao, J., Eugene, T.S., Chandra, P., Steenkiste, P., Zhang, H.: Customizable virtual private network service with QoS. Computer Networks 36, 137-151 (2009)

11. Rossberg, M., Schaefer, G.: A survey on automatic configuration of virtual private networks. Computer Networks (2011)

12. Houidi, I., Louati, W., Ameur, W., Zeghlache, D.: Virtual network provisioning across multiple substrate networks. Computer Networks 55, 1011-1023 (2011)

13. Saaty, T.L.: The Analytic Hierarchy Process. McGraw-Hill, New York (1980)

14. Keranen, A., Ott, J., Karkkainen, T.: The ONE Simulator for DTN Protocol Evaluation. In: SIMUTools '09 2nd International Conference on Simulation Tools and Techniques, Rome (2009)

15. Keranen, A., Ott, J.: Increasing reality for dtn protocol simulations. Tech. Rep., Helsinki University of Technology, Networking Laboratory (2007), http://www.netlab.tkk. fi/tutkimus/dtn/theone/

16. IEEE 802.15 WPAN Task Group 1 (TG1), http: / / www. ieee802.org/15/pub/TG1.html

17. IEEE 802.11 Wireless Local Area Networks, http: //ieee802 .org/11/

18. Spyropoulos, T., Psounis, K., Raghavendra, C.: Spray and Wait: An Efficient Routing Scheme for Intermittently Connected Mobile Networks. In: ACM SIGCOMM Workshop on Delay-Tolerant Networking, WDTN (2005) 